\title{
GEOBIBLINE Y SU COMPARACIÓN CON OTRAS BASES DE DATOS GEOGRÁFICAS
}

Eva Novotná

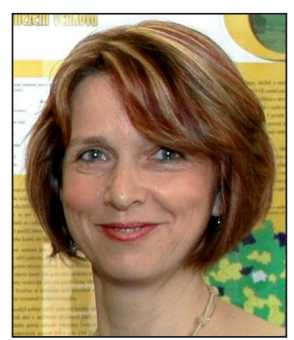

Eva Novotná, doctora en filosofía y en gestión, es directora de la Biblioteca de Geografía y de la Colección de mapas de la Facultad de Ciencias, de la Univerzita Karlova (Universidad Carolina), Praga. Gerente del proyecto Bibliografía geográfica online de la República Checa. Ha trabajado durante más de 20 años en bibliotecas especializadas, 15 de ellos como gerente. Dirige proyectos de automatización de bibliotecas, así como de autoridades y bibliografías. Co-dirige el proyecto Tecnología para el procesamiento de las colecciones de mapas de la República Checa. Autora de artículos, exposiciones especiales, catálogos y del libro Geografía de apoyo a la información (2009) y co-autora del libro Bibliografía geográfica online de la República Checa (2011).

Geografické knihovny, Mapové sbírky Geografická sekce PřF, Univerzity Karlovy Albertov, 6. 12843 Praha 2, República Checa novotn48@natur.cuni.cz

\section{Resumen}

Se describe la creación de la base de datos Geobibline y se compara con otras similares en geografía. Históricamente, las bases de datos tradicionales se crearon con el trabajo bibliográfico metódico llevado a cabo generalmente por una sola institución, y también, en las últimas décadas por bibliotecas que cooperan en un catálogo colectivo. Es el caso que presentamos: gracias la colaboración entre 20 bibliotecas especiales checas se ha creado en sólo 4 años una base de datos que contiene 162.000 registros bibliográficos sobre temas geográficos de la República Checa, inicialmente sólo del siglo 20. Adicionalmente, la colaboración con el proyecto Temap ha permitido ampliar la cobertura temporal hasta el inicio de los libros impresos (1450). La tipología de los documentos extractados es amplia e incluye la literatura gris. A los registros se unen los objetos (mapas, artículos, portadas de periódicos), las direcciones url de los textos completos y también enlaces a Google Books. La base de datos realizada se ha comparado con otras equivalentes de Alemania, Polonia y EUA usando unos encabezamientos de materias concretos, comparables a nivel internacional. La sostenibilidad de Geobibline está garantizada gracias a la importación automática de datos y a su conexión con Temap.

\section{Palabras clave}

Bases de datos bibliográficas, Bibliotecas geográficas, Geobibline, Geodok, Geobib, Bibliografia geografii Polskiej, República Checa.

\section{Title: Geobibline and how it compares with other geographic databases}

\section{Abstract}

The development of the Geobibline database is described. Historically, traditional databases were created using bibliographic methods usually carried out by a single institution, but in recent decades multiple libraries have often cooperated to create joint catalogues. This is the case presented in this article which describes the collaboration of 20 Czech special libraries resulting in the creation of a database that contains 162,000 bibliographic records on geographical topics related to the Czech Republic. The project, completed in just four years, initially covered only 20th century materials, however, collaboration with the Temap project has expanded coverage back to early printed books (1450 A.D.). The typology of the documents referenced is broad and includes gray literature. Records are linked to objects (maps, articles, front pages of newspapers) and there are full text links to Google Books. The database has been compared with counterparts in Germany, Poland, and the United States using internationally comparable specific subject headings. The sustainability of Geobibline is ensured by the automatic import of data and their connection with Temap.

\section{Keywords}

Bibliographic databases, Geographical libraries, Library cooperation, Geobibline, Geodok, Geobib, Polish geographic bibliography, Czech Republic.

Novotná, Eva. "Geobibline y su comparación con otras bases de datos geográficas". El profesional de la información, 2012, mayo-junio, v. 21, n. 3, pp. 304-311. 


\section{Introducción}

El fundador del Instituto Geográfico de la Universidad Carolina (UC) en Praga, Václav Švambera (1866-1939), tenía grandes expectativas sobre las bibliografías geográficas de la República Checa hace ya un siglo (Švambera; Basl, 1911). Era lógico: el trabajo bibliográfico se considera la forma básica de sistema de información de cualquier disciplina académica sólida.

Las bibliografías impresas checas creadas en el campo de la geografía a lo largo del s. XX eran muy dispersas -cronológica, temática e institucionalmente-. Los registros bibliográficos de las bases de datos de las principales bibliotecas, catálogos colectivos en checo o en la bibliografía nacional eran insuficientes (Novotná, 2009).

Un programa del Ministerio de Cultura checo titulado Abrir el acceso y proteger las fuentes culturales, artísticas y científicas ayudó a resolver esta crisis de falta de fuentes de información: entre 2008 y 2011 fue posible crear una base de datos en libre acceso (sin necesidad de licencia) llamada Geobibline (Geografická knihovna et al., 2008-2011), dirigida a especialistas en geografía, profesores, estudiantes, estudiosos y público en general.

Para implementarla se tuvieron en cuenta experiencias tradicionales extranjeras, tales como el repertorio internacional francés Bibliographie internationale géographique (Prodig, 1995-), las bibliografías de Chauncy D. Harris (1975, 1976, 1985), la monografía Geographic information de Jenny M. Johnson (2003) y especialmente las bases de datos bibliográficas geográficas Geodok (del Institut für Geographie, 2011), Bibliografía geografii Polskiej (Gazicka; Peliwo, 2011) y Geobib (American Geographical Society Library, 2011).

\section{Creación de la base de datos}

\subsection{Criterios para la selección de documentos}

Al principio hubo que establecer los criterios de selección. Además de la atención básica a la geografía, el otro criterio fue el aspecto checo, es decir, documentos de autores checos y sobre la República Checa. Se asumió que las bases de datos temáticas en inglés, alemán y polaco cubrirían la mayoría de las revistas con factor de impacto, revistas evaluadas por pares, y otros documentos en esos idiomas, relacionados con Chequia.

\subsection{Secuencia de trabajo}

Se extrajeron los registros del catálogo centralizado de la Universidad Carolina (UC) y se creó Geobibline como base de datos separada. Después de una serie de negociaciones con la Biblioteca Nacional (NK) y el Centro de Computación de la UC (ÚVT UK), se tomó la decisión de importar los registros bibliográficos de los tipos de documentos que no estaban incluidos en la base de datos original de la Biblioteca geográfica o lo estaban sólo parcialmente. Los primeros fueron los documentos cartográficos de la NK-al ser depositaria del depósito legal la colección de estos documentos era la más grande y completa (en 2008 contenía 6.000 registros cartográficos relacionados con Chequia)-. Además se importaron artículos de temas geográficos de la bibliografía nacional realizada por la $N K$ desde mediados de los 90 . Pos- teriormente se copiaron los registros de monografías, documentos electrónicos, tesis y revistas de la Biblioteca de Geografía de la Facultad de Ciencias de la Universidad Carolina (FCUC) a la base de datos Geobibline.

Para llevar a cabo las búsquedas online en las bases de datos de la NK, a principios de 2008 el ÚVT UK prestó un cliente de interfaz gráfica que almacena los registros seleccionados en el servidor de la $N K$, desde donde se envían al servidor ÚVT UK. Las búsquedas fueron realizadas utilizando encabezamientos de materia en combinación con el lugar de publicación y el tipo de documento.

En 2009 se hicieron búsquedas adicionales en la NK, inicialmente con un proceso semi-automático, que más tarde fue automatizado por completo. Las bibliografías originales empezaron a recopilarse en las bibliotecas participantes en 2008. Otros tipos de documentos se exportaron de forma automática todos los días desde la Biblioteca de Geografía en la FCUC identificados a través del campo <GEO>. La primera vez los registros de las otras bibliotecas se importaron offline pero desde 2011 la importación ha sido totalmente automatizada a intervalos regulares, no sólo de la $N K$, sino también de la Biblioteca de Moravia, las bibliotecas de la Academia de Ciencias, bibliotecas de universidades, científicas estatales y otras organizaciones profesionales. Cada mes la base de datos aumenta en varios cientos de registros procedentes de fuentes externas. A principios de 2012 la base de datos Geobibline contenía 162.000 registros bibliográficos, con un incremento anual de alrededor de 20.000 registros.

\subsection{Metodología}

La metodología básica para la creación de la base de datos fue elaborada y actualizada de forma progresiva. Los registros siguen las reglas de catalogación nacionales e internacionales, fijándose el nivel mínimo del registro MARC21. Se utilizaron encabezamientos de materia en checo. Para los participantes en el proyecto se creó una interfaz y un acceso a la base de datos a través del protocolo Z39.50.

\subsubsection{Método del campo atributo GEO}

Hubo que resolver un problema fundamental derivado del trabajo simultáneo en dos bases de datos (el catálogo centralizado de la UC y Geobibline): había que catalogar los documentos una sola vez, al mismo tiempo que se exportaban (preferiblemente de forma automática) a la otra base de datos. Al final, se optó por introducir atributos en los campos como la solución más simple. De acuerdo con el atributo <GEO> predefinido en el catálogo de la sub-biblioteca de geografía de la FCUC, se identificaba el registro bibliográfico y se exportaba automáticamente del catálogo centralizado de la UC a la base de datos Geobibline.

\subsubsection{Comprobación de duplicados}

El problema de los registros duplicados comenzó a resolverse para los registros de monografías recién importados y para los nuevos registros cartográficos que las bibliotecas participantes podrían haber enviado más de una vez.

Antes de la primera importación era necesario tener ya resuelto el tema de los registros duplicados. En el sistema de bibliotecas Aleph de la UC, la comprobación automática se 


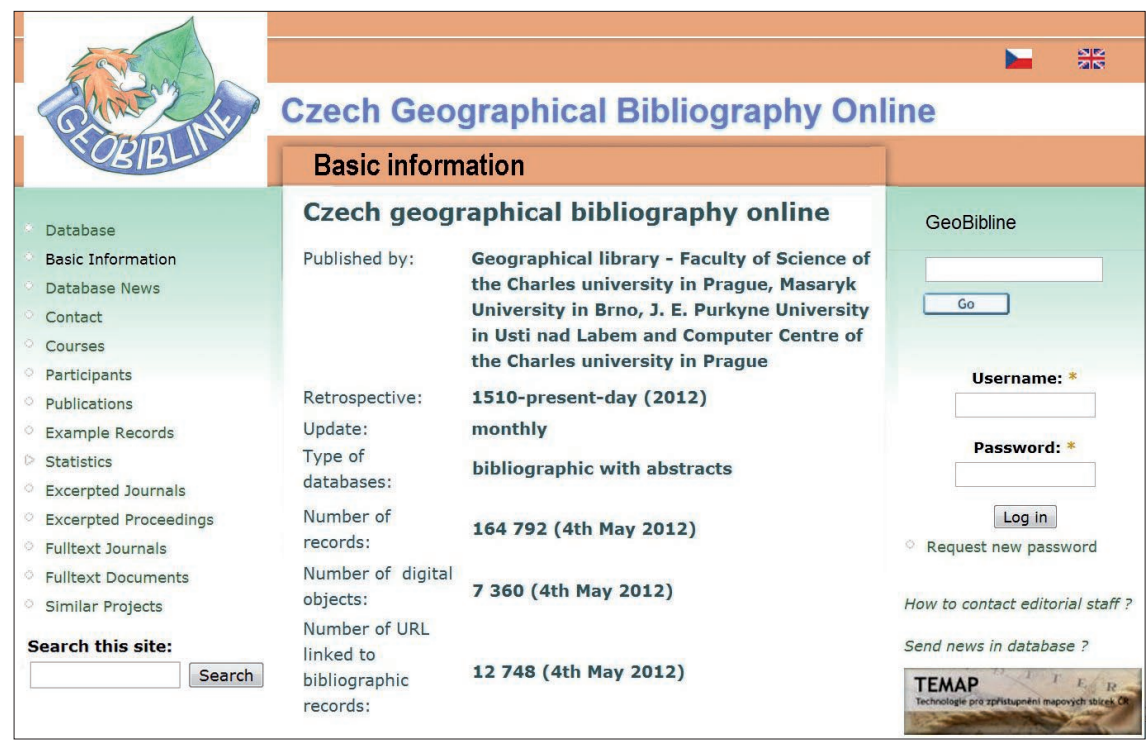

http://www.geobibline.cz

hizo utilizando al menos dos campos de control y el campo $<001>$ (MARC21). El sistema tenía que marcar los registros duplicados e informar de los errores, a continuación el administrador de la base de datos marcaba los duplicados reales y el sistema posteriormente terminaba importando los registros. El proceso se depuró y automatizó con éxito. Un correo electrónico automático informa de todos los registros duplicados encontrados (Novotná, 2011).

\subsubsection{Textos completos}

Desde el principio la base de datos Geobibline fue entendida como un sistema que en el futuro daría acceso a los textos completos. La mayoría de los editores estuvieron de acuerdo, aunque no tienen demasiada experiencia en tratar el asunto de los derechos de autor, y en muchos casos no existen contratos entre los editores y los autores.

Un problema era la inexistencia de textos completos electrónicos, pero la situación cambió pronto al comenzar muchas editoriales a publicar electrónicamente.

\section{Licencias}

Los derechos de autor y los temas legales fueron tratados de forma gradual. Se empezó a negociar con los propietarios de derechos de autor que ya habían expuesto sus documentos en internet, preguntándoles sobre la posibilidad de enlazar a los textos completos de su web. Más tarde pedimos al representante legal de la editorial o de la institución científica que había publicado el documento su consentimiento por escrito para guardar los datos en el repositorio de la Facultad y publicarlos a través de la base de datos de Geobibline. Con el tiempo empezamos a firmar contratos de licencia continua de provisión de ficheros electrónicos de los textos completos. Los contratos también incluyen la autorización de la editorial de hacer accesible y tener en la base de datos Geobibline todos los volúmenes publicados con anterioridad. Se respeta la política editorial de cada revista en particular, especialmente en relación al embargo por un período de tiempo específico.

La información sobre copyright, modificada a petición del titular de los derechos de autor, se incluye en el registro bi- bliográfico en el campo 500 del MARC 21. El usuario siempre está informado acerca del copyright, pues antes de abrir el texto completo puede leer lo siguiente:

"Advertencia sobre restricciones definidas por la Ley de Derecho de Autor (Ley N. 121/2000 Coll.). Bajo las condiciones definidas por la Ley de Derecho de Autor, las bibliotecas y archivos están autorizados a realizar libremente fotocopias u otras reproducciones de trabajos de autor. La condición específica es que esas fotocopias $u$ otras reproducciones no pueden ser utilizadas para otros propósitos que no sean el estudio privado o la investigación. Si un usuario solicita un documento y realiza una fotocopia o reproducción en conflicto con las condiciones definidas por la Ley de Derecho de Autor, será responsable de infracción de copyright. Esta biblioteca se reserva el derecho de rechazar un pedido de copias, si en su opinión el cumplimiento de dicho pedido supondría una infracción de copyright" (Información de copyright en Geobibline).

Además de los artículos, los textos completos de las tesis académicas también van siendo accesibles. La base de datos proporciona acceso a las tesis de la Universidad Masaryk, de Brno y de la UC. El usuario externo tiene que registrarse para poder acceder a los textos completos. Aquí también se le informa sobre las condiciones de la licencia:

"Toda la información obtenida mediante la búsqueda en las tesis publicadas por la UC de Praga, no puede ser usada con ánimo de lucro ni ser declarada como estudio, investigación o actividad creativa de otra persona que no sea el autor" (condiciones de licencia del repositorio de la UC).

La Biblioteca Nacional ha elaborado un contrato para el suministro de recursos online, de acuerdo con el cual los proveedores pueden dar el consentimiento a la biblioteca para que los conserve permanentemente y dé acceso a los mismos. Este contrato se tomó como modelo para Geobibline. También se redactó un modelo de contrato de licencia para los autores (o sus herederos propietarios de los derechos) con el fin de obtener su consentimiento para la publicación. En la República Checa los derechos expiran a los 70 años de la muerte del autor. Teóricamente es posible publicar sin permiso expreso obras de autores fallecidos antes de 1942, pero aun así pueden surgir obstáculos legales.

Con el Aleph digital asset module (ADAM) se enlazaron a las referencias bibliográficas los textos completos de (principalmente) artículos, portadas de revistas o muestras especiales de manuscritos. ADAM es un módulo especial del software Aleph 500 que permite integrar objetos digitales con sus metadatos (fichas descriptivas) en el entorno de la biblioteca. Los 7.360 objetos digitales adjuntos (mayo de 2012) se pueden buscar por su texto completo y luego visualizarse (ÚVT, 2008). 
Otra opción es conectar los artículos disponibles online mediante sus urls en el campo 856 de MARC. Desde 2010 se realizan actualizaciones y controles periódicos de urls rotos mediante una función de Aleph. En mayo de 2012 la base de datos contiene más de 12.700 direcciones url.

\subsection{Cooperación entre bibliotecas}

El proyecto se inició en la Biblioteca de Geografía de la FCUC, de Praga, en la biblioteca de la Universidad Masaryk, de Brno y en el Departamento de Geografía de la Facultad de Ciencias, Universidad J. E. Purkyne, de Usti nad Labem. Las dos primeras bibliotecas trabajaban con el software Ale$p h$, por lo que la exportación de datos fue fácil de configurar. La tercera biblioteca utiliza el software Rapid Library y la situación fue mucho más complicada. Tomó mucho tiempo depurar las importaciones.

Poco a poco fue creciendo el interés por parte de otras bibliotecas, hasta un total de 20. En la práctica esto significa que las "grandes" bibliotecas (Nacional, Academia de Ciencias, estatales de investigación, etc.) suministran registros bibliográficos a las bibliotecas más pequeñas y especializadas. Para compartir los registros se tuvieron que solventar varias dificultades debidas a las diferentes opciones de catalogación e indexación en diversos campos MARC locales.

Con la Biblioteca Nacional no hubo problemas técnicos porque trabaja con Aleph, pero sí los hubo con las pequeñas bibliotecas que disponen de sistemas diferentes como ISIS, $K P$-Win, etc. Algunos no soportan el formato MARC 21, sólo Unimarc. A pesar de que se trabajara con normas e instrucciones comunes, había diferencias. A menudo surgían "restos" de otros sistemas más antiguos, así como prácticas de catalogación locales (por ejemplo, autoridades locales que no estaban vinculadas a las nacionales). Algunas bibliotecas no utilizaban encabezamientos de materias, y a veces los registros importados eran más difíciles de corregir que si se hubieran creado nuevos.

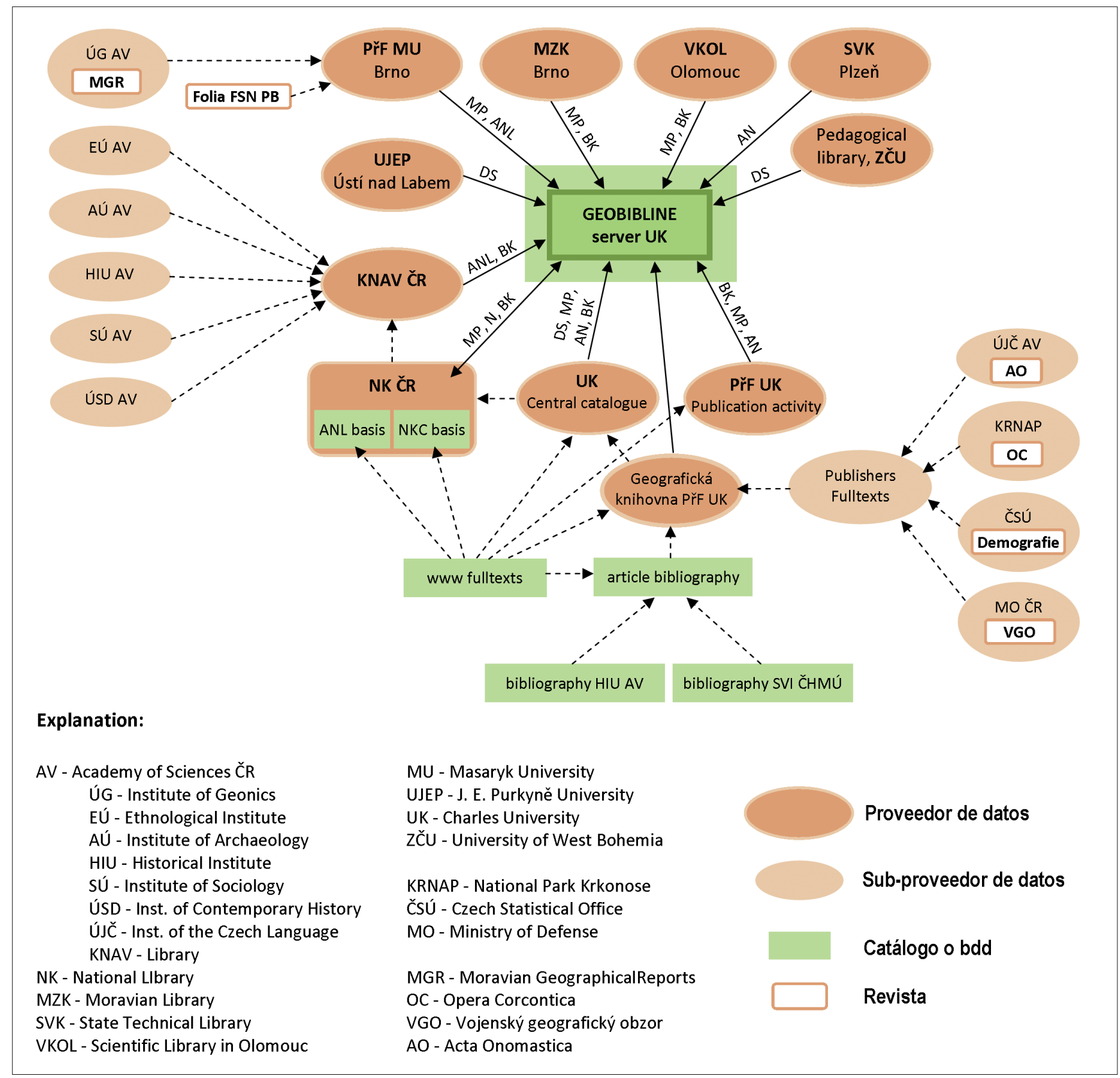

Figura 1. Modelo de proceso de Geobibline 
Pero no sólo se importaron registros de los clásicos catálogos de biblioteca. El gran problema fue la importación de los registros de las llamadas "actividades de publicación" de la Universidad Carolina que realiza su personal, y que no se incluyen normalmente en el catálogo central. Los registros los habían hecho los mismos autores y estaban llenos de errores. La importación requirió mucho trabajo manual, pero el contenido valía el esfuerzo.

Finalmente, la propia Biblioteca Nacional extrajo enlaces url de Geobibline para su catálogo. Y también las bibliotecas participantes recibieron feedback sobre su catalogación con los errores encontrados.

La base de datos Geobibline ha introducido un nuevo modelo de colaboración en el panorama de las bibliotecas checas. Las grandes bibliotecas con colecciones que abarcan por lo general una amplia gama de temas que van desde las humanidades a la tecnología han acordado colaborar estrechamente con las pequeñas bibliotecas de investigación, en su mayoría especializadas en geografía.

\section{Acceso de los usuarios}

La base de datos se puede consultar online y en DVD. La página principal del opac ofrece entrada tanto a Geobibline como a otras bases de datos de instituciones colaboradoras. Se ha creado una versión de consulta en inglés y muchos documentos poseen términos en este idioma, especialmente los trabajos de la universidad.

Los usuarios pueden visualizar, almacenar, enviar por correo-e o localizar los documentos, ya sea en el Catálogo Colectivo de la República Checa, en la NK, la Biblioteca de Moravia en Brno, la Biblioteca de Investigación de Olomouc, o en la Biblioteca del Congreso.

En 2010 se puso en funcionamiento un servicio de gestión de referencias de la base de datos Geobibline que las formatea según ISO 690 e ISO 690-2 (Krčál, 2004-2011):

http://www.citace.com

\section{Comparativa de Geobibline con otras bases de datos internacionales}

Hacemos la comparación con otras bases de datos geográficas gratuitas de Polonia, Alemania y Estados Unidos. No se ha hecho con bases de datos comerciales como GeoRef, GeoBase y otras que tienen restricciones de uso.

\subsection{Bibliografia Geografii Polskiej (BGP)}

La Bibliografía geográfica de Polonia se publica en la Academia de Ciencias de Polonia en Varsovia y ha estado disponible en forma impresa desde 1956.

En internet está desde 1999 (GazickaWójtowicz; Peliwo, 2011), cubriendo el período 1985 a 2006 (lleva, pues, un considerable retraso de producción), y funciona con el software ISIS. Contiene 57.203 registros, que a su vez ofrecen información de unos 150.000 títulos de artículos de revista, capítulos de libro, ponencias, mapas y atlas. El crecimiento anual es de 2.5003.000 registros (Gazicka-Wójtowicz, 2012).

Incluye publicaciones educativas, científicas y populares de geógrafos polacos y artículos geográficos de toda índole. Indiza 150 títulos de revistas polacas y extranjeras.

Los editores son Dorota Gazicka y Jan Peliwo.

La Academia de Ciencias de Polonia mantiene un repositorio con 1.036 objetos digitales:

http://rcin.org.pl/igipz

que también aloja al Repozytorium Cyfrowe Instytutów Naukowych (repositorio de 16 institutos científicos), que contiene 5.000 publicaciones (mayo de 2012):

http://rcin.org.pl

\section{Comparación entre BGP y Geobibline}

Los visitantes no polacos pueden echar en falta que haya poca información básica en inglés sobre la base de datos, pues sólo se ofrece un pequeño manual en este idioma. La cobertura temática de la bdd polaca es mucho más amplia que la de Geobibline, lo cual se debe a la historia y a los recursos humanos de sus respectivas instituciones. En cambio los registros más antiguos de la polaca son sólo de 1980.

La búsqueda en ambas es bastante similar, aunque el software ISIS impone serias limitaciones.

Una vez seleccionados los resultados, hay una opción para ver o imprimir los registros en cuatro tipos de formatos, pero no pueden ser salvados o enviados por correo electrónico. No acepta el formato MARC o la norma ISO 690.

\section{Dorota Gazicka, Jan Peliwo \\ Bibliography of Polish}

Geography since 1985

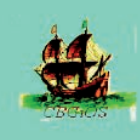

The database has been created by Central Library of Geography and Environmental Protection of the Institute of Geography and Spatial Organization Polish Academy of Sciences

c) Central Library of Geography and Environmental Protection | more info about database List of indexed journals $\mid$ User's guide $\mid$

Software Expertus WWW for CDS/ISIS databases @ SPLENDOR, Poznań

In fields '... - any words' type serveral words or word roots nearby; example

A term can be selected from the index: example

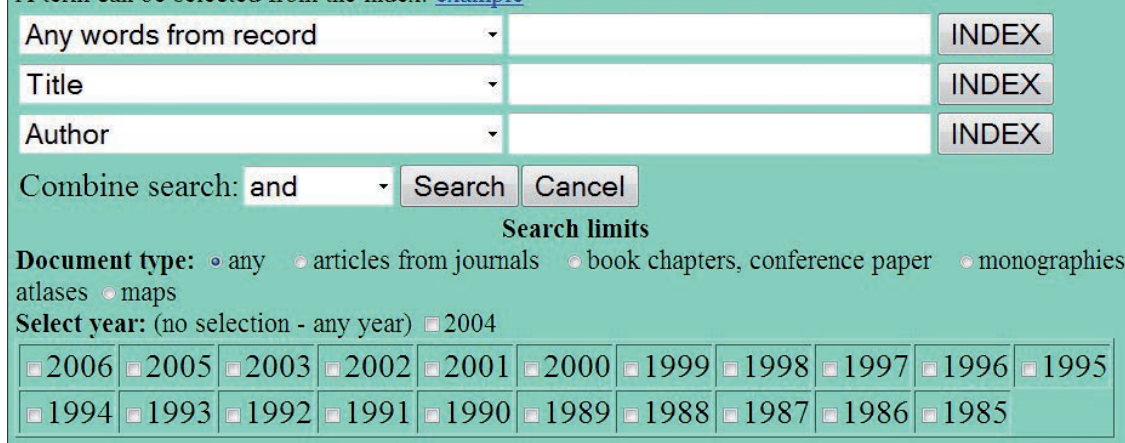

Search resources of other libraries: (no selection - all libraries from list; other localization given if and http://www.cbgios.pan.pl/bazy/bgp 


\subsection{Geodok}

La bdd geográfica bibliográfica Geodok 5.0 es producida por el Institut für Geographie, de la Universität Erlangen, en Nurenberg, en colaboración con la Asociación de Geógrafos de las Universidades Alemanas. Las referencias de los documentos más antiguos datan de 1950, pero la mayoría son posteriores a 1980. Se puso en funcionamiento en 1999 y se actualiza mensualmente. Contiene 153.000 referencias $(75 \%$ de artículos y $25 \%$ de monografías), con un crecimiento anual de 5.000 (Huber, 2012; Institut für Geographie, 2011). Usa el software Aleph.

Indexa cerca de 500 revistas geográficas en alemán, inglés y francés. Los usuarios realizan unos 3 millones de búsquedas anuales en la versión en inglés (Novotná, 2009).

Los resultados se muestran en un formato corto. Los encabezamientos de materia, un enlace al texto completo en ZDB-OPAC, Elektronische Zeitschriftenbibliothek, y el servicio SFX de resolución de enlaces están claramente situados a la derecha de cada registro. Los resultados se pueden seleccionar, imprimir, convertir a pdf, guardar o enviar por correo electrónico. Geodok también permite exportar los resultados a Citavi y EndNote. Existe una fuente RSS que se puede utilizar para enviar consultas de los usuarios.

\subsection{Geobib}

Está publicada por la American Geographical Society Library de la University of Wisconsin-Milwaukee, EUA (American Geographical Society Library, 2011), y abarca desde 1985 hasta diciembre de 2005, fecha en la que dejó de actualizarse. Contiene 115.000 registros que siguen siendo accesibles (Peschel, 2012).

Tenía el equivalente impreso Current Geographical Publications que terminó en 2003, y desde entonces sólo continuó online (ProQuest, 2011). Funciona con Aleph.

Los registros están indexados mediante el catálogo de materias de la American Geographical Society Library.

\subsection{Estructura temática}

Para comparar los contenidos de las bases de datos se seleccionaron cuatro áreas temáticas:

- Geografía física: geología, geomorfología, meteorología, climatología, hidrología, edafología, biogeografía y ecología;

- Geografía social/humana: geografía social, económica, del transporte, cultural, política, regional y sociología;

- Demografía;

search by using the first input

box!

Barcelona

e.g. Munich City*

AND - Keyword (Titlk -

e.g. Munich City*

AND $\cdot$ Author/Edito

e.g. Richter, M. or Richter

AND - Year

e.g. 1995 od. $1990-2000$

Search Reset Help

\section{GEODOK Geographic Literature Database}

Configuration | Help

Quick

Search

http://www.geodok.uni-erlangen.de

e.g. Bätzing Alpen "Geographische Rundschau" (max. 5 search

terms)

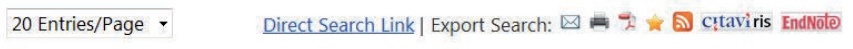
add to Selection List $\quad$ First Previous 12 Next Last (31 Entries)

Charnock, G. Ribera-Fumaz, R. (2011) Urban development | Urban planning | Catalonia A new space for knowledge and people? Henri Lefebvre, representations of space, and A

In: Environment and Planning / D Volume: 29 Issue: 4 Page: 613-632

In: Environment and Planning / D

Martori, J. Apparicio, P. (2011) ZDB-OPAC EZB-Fulltext OSFX

Spain | Migration | Metropolitan are

Changes in spatial patterns of the immigrant population of a southern European

metropolis. The case of the Barcelona metropolitan area (2001 - 2008)

In: Tijdschrift voor Economische en Sociale Geografie Volume: 102 Issue: 5 Page: 562-581 Article Erlangen holding: $12 \mathrm{GG} / \mathrm{Z} 554$ ZDB-OPAC EZB-Fulltext $\mathrm{OSFX}$

Pallares-Barbera, M. Badia, A. Duch, J. (2011) Spain | Urban planning | Urban development Cerda and Barcelona. The need for a new city and service provision

In: Urbani izziv Volume: 22 Issue: 2 Page: 122-136 Article Erlangen holding: $12 \mathrm{GG} / \mathrm{Z} 590$ ZDB-OPAC EZB-Fulltext四 OSFX

- Disciplinas auxiliares: cartografía, sistemas de información geográfica (SIG), teledetección y estadísticas (Raska, 2011).

Podría plantearse la objeción de que existe un gran número de definiciones de geografía, la descripción de la temática de los documentos es subjetiva, y la indización varía en bases de datos diferentes: a veces es más profunda, y otras veces parece ser superficial. Esto apareció con el título general de geografía social/humana, por ejemplo, con sólo 30 documentos clasificados por este concepto en la base de datos de Polonia. Es probable que existan muchos más documentos con descripciones más detalladas.

El gráfico 1 con las disciplinas básicas geográficas que fueron seleccionados inicialmente ofrece la comparación final. La bdd checa tiene la mayor incidencia de geografía física

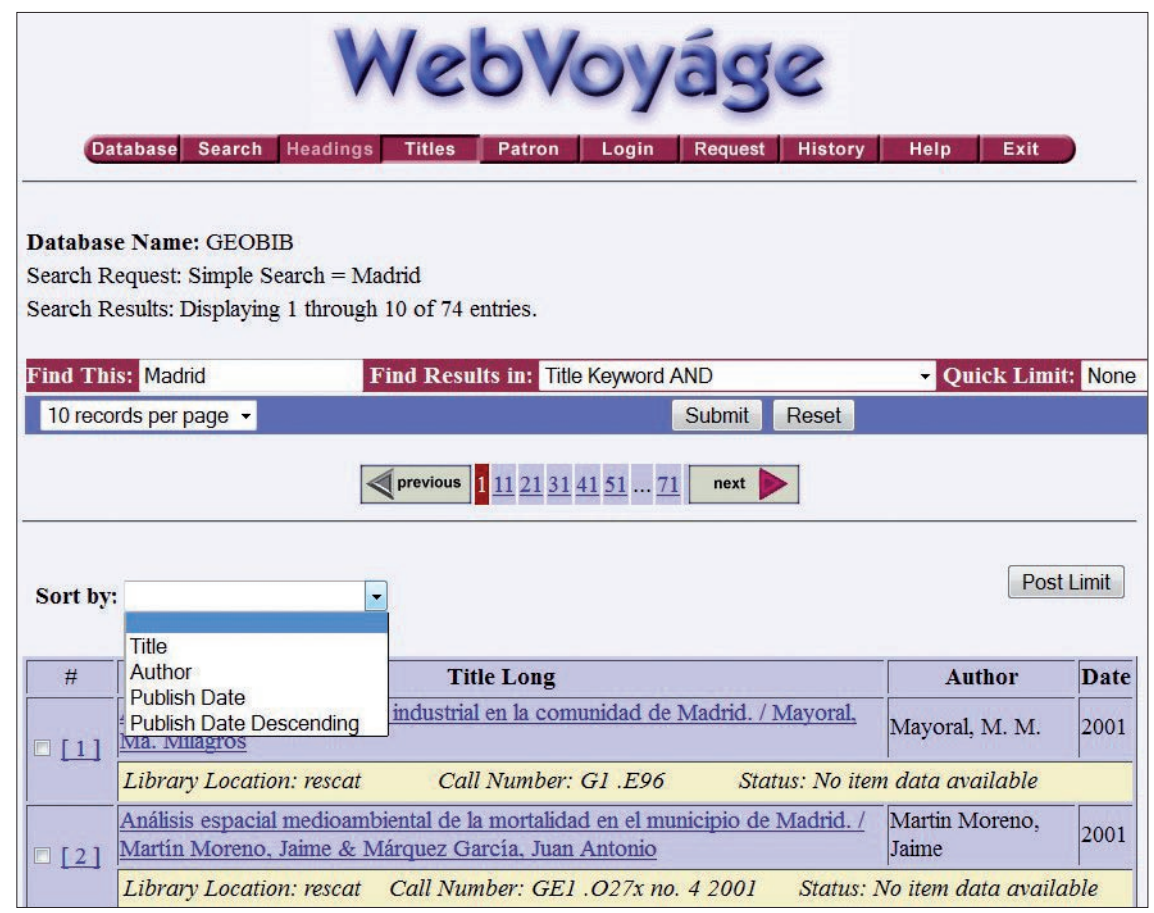

http://geobib.lib.uwm.edu 


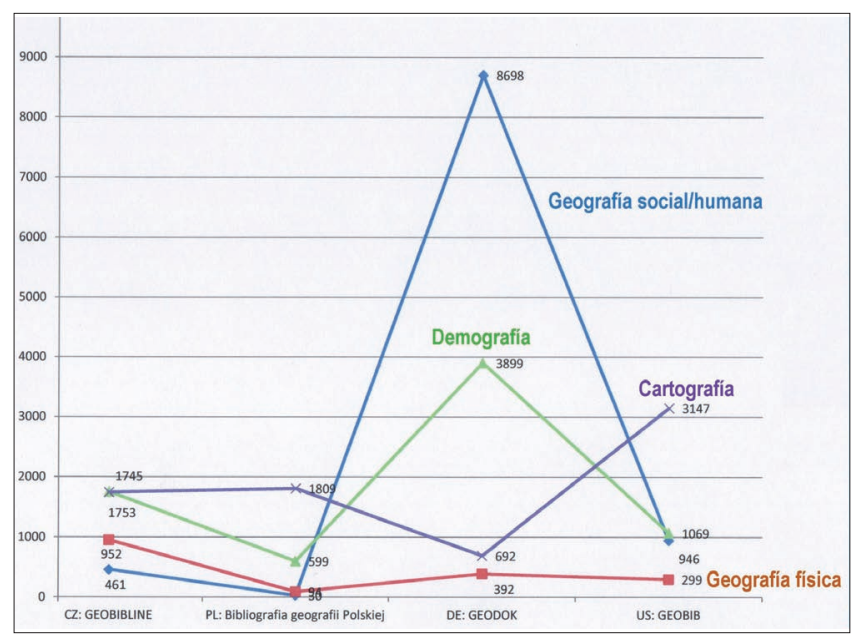

Gráfico 1. Gráfico comparativo de los encabezamientos de materia de geografía social, geografía física, demografía y cartografía en las cuatro bases de datos geográficas internacionales

(952), la alemana es mejor en geografía social (8.698) y demografía (3.899), y la norteamericana es mejor en cartografía (3.147). A partir de esta comparación, la Geodok alemana podría coronarse como "reina" de las bases de datos geográficas.

\section{Conclusión}

La base de datos Geobibline es ya comparable con los productos extranjeros similares. Se veloz método de creación en sólo 4 años es un aspecto especialmente interesante (la de EUA lleva 25 años, y la polaca y la alemana llevan 13). Esto fue posible gracias a la colaboración entre 20 bibliotecas. Entre ellas las exportaciones de datos no sólo van de arriba hacia abajo sino también de abajo hacia arriba.

\begin{tabular}{|l|c|c|}
\hline \multicolumn{1}{|c|}{ Nombre } & No de registros & Período \\
\hline Geobibline & 164.000 & s. XVI - actual \\
\hline Bibl. Geografii Polskiej & 58.000 & 1985 - 2006 \\
\hline Geodok & 152.000 & $1950 / 1980$ - actual \\
\hline Geobib & 115.000 & $1985-2005$ \\
\hline
\end{tabular}

Poder trabajar con el software Aleph (bdds de Estados Unidos y de la República Checa) permite ciertas funciones que no son posibles con la polaca y la alemana, las cuales ofrecen menos opciones.

Una ventaja de la bdd checa son los adjuntos: objetos y textos completos de los documentos. La alemana también ofrece textos completos a través de la Elektronische Zeitschriftenbibliothek (EZB) en Ratisbona (Regensburg).

Geobibline llena un vacío sobre información geográfica en Centroeuropa, como lo prueba el número de visitas que recibe: 877.000 desde que se puso en funcionamiento en 2009. El mayor interés procede de Estados Unidos, Países Bajos y Alemania.

El proyecto Temap (Tecnología para el acceso a las colecciones de mapas checos), que ha colaborado en la bdd desde 2011, va a incluir más documentos antiguos en texto completo.

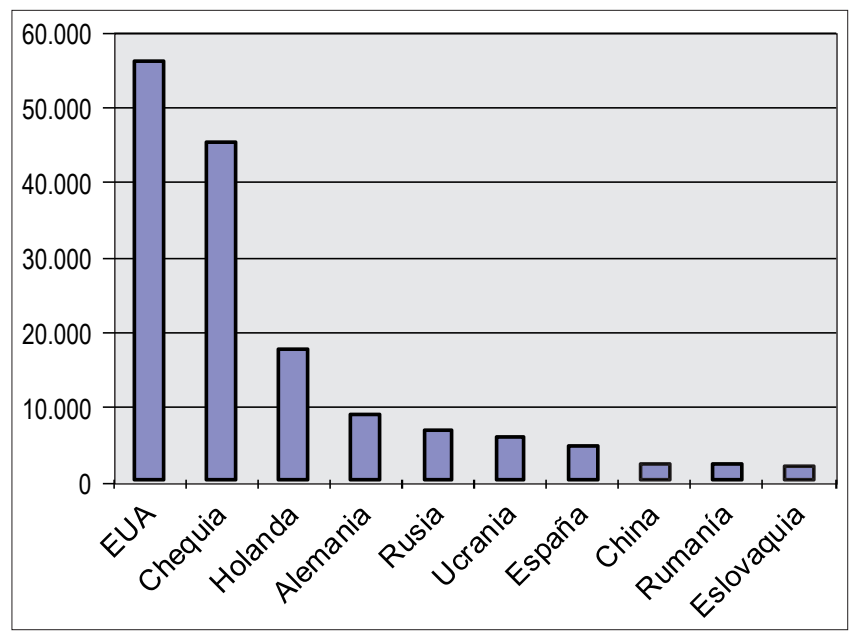

Gráfico 2. Tráfico por países del sitio www.geobibline.cz en 2011

Las bdds geográficas descritas se complementan entre sí, y se distinguen en la tipología de documentos, y en la forma en que son elaboradas. Cada base de datos tiene sus fortalezas y debilidades. En el futuro, sería sin duda beneficioso para los usuarios que se pudieran consultar las cuatro desde un portal único, pero habría que resolver la barrera del idioma. Los documentos de las europeas tendrían entonces que indexarse también con palabras clave en inglés, o habría que usar un diccionario geográfico común multilingüe. Después, a los usuarios se les ofrecería la oportunidad de elegir entrar en una única base de datos cada vez, o consultarlas todas al mismo tiempo. ¿Qué elegirían?

\section{Referencias}

American Geographical Society Library. Geobib.

http://geobib.lib.uwm.edu/cgi-bin/Pwebrecon.cgi? $D B=$ local\&PAGE=First

\section{Elsevier. Scopus.}

http://www.scopus.com/home.url

European Science Foundation, ERIH.

http://www.esf.org/research-areas/humanities

Gazicka-Wójtowicz, Dorota; Peliwo, Jan. (ed.). Bibliografia geografii Polskiej.

http://www.cbgios.pan.pl/bazy/bgp

Gazicka-Wójtowicz, Dorota. About Bibliografia geografii Polskiej 1985+ (email), 2012.

Geografická knihovna...[et al.]. Geobibline. http://www.geobibline.cz

Harris, Chauncey D. (ed.) Guide to geographical bibliographies and reference works in Russian or on the Soviet Union. Chicago: University of Chicago, 1975.

Harris, Chauncey D. (ed.) Bibliography of geography. Chicago: University of Chicago, 1976.

Harris, Chauncey D. et al. (ed.) A geographical bibliography for American libraries, Washington, D.C.: Association of American Geographers, 1985. ISBN: 089291193 X

Hüber, Thomas. About Geodok (email), 2012. 
Johnson, Jenny-Marie. Geographic information. Westport, CT: Greenwood Press, 2003. ISBN: 1573563927

Institut für Geographie. Geodok.

http://www.geodok.uni-erlangen.de

Krčál, Martin. Citace 2.0.

http://www.citace.com

Národní knihovna ČR. ANL.

http://aleph.nkp.cz/F/VI1TUHH6KFAK894RTK7DV3RC7IE

KNVKD28JEHI7E2LKTLP8BTL-35362 ?func=file \&file_ name $=$ base-info

Novotná, Eva. Informační podpora geografie. Praha: Univerzita Karlova, Př́rodovědecká fakulta, 2009. ISBN: 97880 86561424

Novotná, Eva, et al. Geografická bibliografie ČR on-line. Praha: Vydavatelství VŠCHT, 2011. ISBN: 9788070807736

Peschel, Susan. About Geobib (Email), 2012.
Prodig. Bibliographie géographique internationale, v. 100, tome 1 (1995-).

ProQuest. Ulrichsweb: the global source for periodicals. http://www.ulrichsweb.com/ulrichsweb/default.asp?nav Page $=4 \& \#$

Rada pro výzkum, vývoj a inovace. Seznam recenzovaných neimpaktovaných časopisů vydávaných v ČR.

http://www.vyzkum.cz/FrontClanek.aspx?idsekce $=495942$

Raška, Pavel. "Projekt Geobibline a e-learning v geografickém vzdělávání." In: Novotná, E. et al. Geografická bibliografie ČR on-line. Praha: Vydavatelství Všcht, 2011, pp. 122. ISBN 9788070807736

Švambera, Václav; Basl, Antonín (ed.). Bibliografie české literatury geografické, v. I., 1906 a 1907. Praha: Geografický ústav České university, 1911.

ÚVT. ADAM.

http://aleph.cuni.cz/ALEPH-50.html

\section{Máster en Buscadores: Marketing} y posicionamiento. Modalidad online. Ponte a punto

Recuperación de la información, marketing online innovador, programas de vigilancia tecnológica y de inteligencia competitiva

Dirección: Cristòfol Rovira

Calendario: diciembre 2012 - junio 2013

Más información: www.idec.upf.edu/mbusol

\section{PONTE A PUNTO}

Másters y Programas de postgrado en Comunicación y Ciencias Sociales

Universitat Pompeu Fabra. www.idec.upf.edu cid

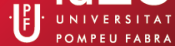

ÁREAS DE FORMACIÓN: Comunicación y Periodismo • Derecho • Gestión Cultural y Humanidades • Comunicación y Medios Audiovisuales • Política y Sociedad • Publicidad y Reputación Corporativa • Lenguaje y Traducción • Creatividad en Medios Digitales • Salud y Bienestar Social • Innovación y Gestión de la Información 